\title{
Influence of metal inserts with microformed edges on subsequent injection assembly moulding for media tight electronic systems
}

\author{
Philipp Frey ${ }^{1, a}$, Martina Heinle ${ }^{2}$, Dietmar Drummer ${ }^{2}$, and Marion Merklein ${ }^{1}$ \\ ${ }^{1}$ Institute of Manufacturing Technology, Friedrich-Alexander-Universität Erlangen-Nürnberg (FAU), \\ Egerlandstr. 13, 91058 Erlangen, Germany \\ ${ }^{2}$ Institute of Polymer Technology, Friedrich-Alexander-Universität Erlangen-Nürnberg (FAU), \\ Am Weichselgarten 9, 91058 Erlangen, Germany
}

\begin{abstract}
Recently, there is rising demand of electronic systems with functional density for industrial and automotive applications. Those components, which are typically manufactured by overmoulding blanked metal inserts within an assembly injection process, are often exposed to rough operating conditions. Especially penetrating water can lead to a severe damage of electronic systems which are often crucial to safety. Leakage can be caused among other things by crack initiation within the polymer at sharp edges of the metal insert as a consequence of stress concentration. In order to reduce stress concentration the effect of metal inserts with rounded edges and the forming process to manufacture such inserts is investigated. Since typical sheet thicknesses for electronic components are $1 \mathrm{~mm}$ and less the dimensions of the rounded edges are on the scale of micro features. The microforming operation of rounded edges is provided by open coining. The influence of varying part dimensions is investigated using FE-simulation. Furthermore, ideal rectangular insert shapes are compared to parts with sheared edge geometry. In addition the effect of rounded edges on stress distribution of overmoulded parts is analysed by combining resulting geometries of the forming simulation with the numerical analysis of stress distribution within the polymer.
\end{abstract}

\section{Introduction}

Recently, the automotive and industrial sector are the driving forces behind a rising demand of electronic systems which is due to the increasing degree of automation in production and steady expanding requirements regarding security, emission control and comfort in today's cars. This trend will gain in relevance due to the assumption that by 2017 electrical components will account for more than 30\% of automotive production cost [1]. Those electronic systems are characterized by a high functional density and therefore different materials with particular properties are combined for example metals and polymers [2]. Electronics systems for example engine control units and parts of the break system

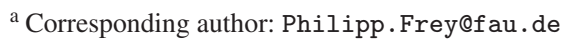

This is an Open Access article distributed under the terms of the Creative Commons Attribution License 4.0, which permits unrestricted use, distribution, and reproduction in any medium, provided the original work is properly cited. 


\section{MATEC Web of Conferences}

Stress Concentration

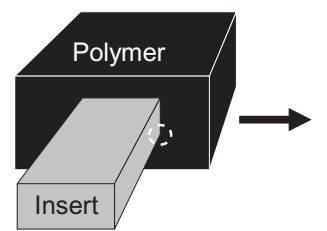

Uniform Stress Distribution

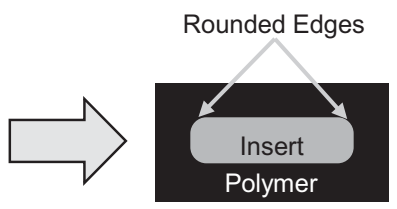

Figure 1. Schematic illustration of crack initiation and the approach to reduce stress concentration with rounded edges

are often mounted at the place of action. Thus, electronic devices have to withstand rough operating conditions like high temperatures, dust, moist and mechanical vibration [3]. Especially penetrating moist can cause serve damage to the device or result in a total breakdown. Given the fact that electronic systems are used in devices which are crucial for safety, like the break system, providing reliable electronic components is of great importance. The combination of processing metal within blanking and forming operations and a subsequent assembly injection moulding process is an efficient way of producing polymer metal hybrids. However, several challenges need to be solved to achieve media tight components. One is that the different material properties lead to crack initiation at sharp edges of the metal insert. Such cracks, which are illustrated in Fig. 1, are a consequence of the shrinkage of the polymer and thermo-mechanical stress [4]. Using rounded edges for achieving a more uniform stress distribution is a promising approach for the reduction of stress concentrations. Since edge rounding by coining can be implemented in a progressive die it is also potentially well applicable for high productive processes.

Considering that metal inserts are typically manufactured by blanking the sheared edges differ from the ideal shape due to rollover, fracture and burr. Hence, an edge rounding process has potential to shape a more regular cross section and can also improve the mechanical properties. This was shown for blanked steel parts with a thickness of $4 \mathrm{~mm}$ and formed edge radii between $1.25 \mathrm{~mm}$ and $2.25 \mathrm{~mm}$ [5]. In contrast, the scope of this paper is to form radii of the edges in the submillimetre range and therefore this process can be assigned to microforming [6]. For that reason aspects like workpiece tolerance gain more relevance. In the case of blanked parts the influence and accuracy of the sheared edge have to be considered as well, since the dimension of rollover and edge radius are in a similar range. Furthermore, the final shape of the insert is of relevance in regard to a subsequent injection moulding process and the stress distribution within the polymer. Edge rounding by forming has the potential to be efficient way to reduce the risk of crack formation since it can be integrated as an additional stage in a progressive die. Thus, it is necessary to understand the relation of different parameters on the forming results in order to implement an edge rounding process for metal inserts for electronic applications.

\section{Experimental setup}

In order to form rounded edges an open coining process is used. It is characterised by two identical punches where the upper punch moves downwards and applies the punch force and the edges are formed simultaneously as it is shown in Fig. 2a. The metal insert is positioned by two locating pins to realise conditions comparable to a progressive die. The punches illustrated in Fig. $2 \mathrm{~b}$ were made out of tool steel 1.3343 hardened to $62 \mathrm{HRC}$. Thus, they were machined by the AgieCharmilles CUT 2000 wire EDM with a surface roughness of $R_{a} 0.81$. The metal inserts with a nominal width of $5 \mathrm{~mm}$ and $1 \mathrm{~mm}$ thickness were produced by laser cutting with the Trumpf TruLaser Cell 7020 and by blanking. The inserts were blanked with a relative die clearance $\mathrm{c}_{\mathrm{rel}}=5 \%$. As workpiece material CuSn6 and AlMg3 
a)

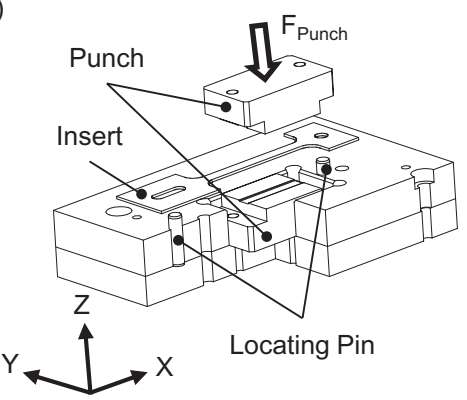

b)

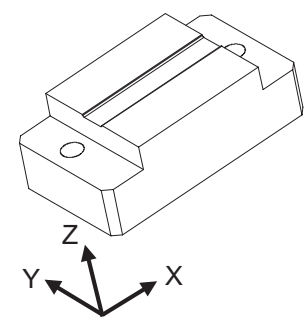

c)

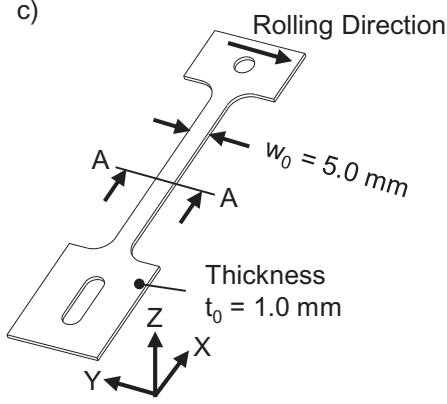

Figure 2. Experimental forming setup (a), exemplary punch with cavity (b) and untreated metal insert (c).

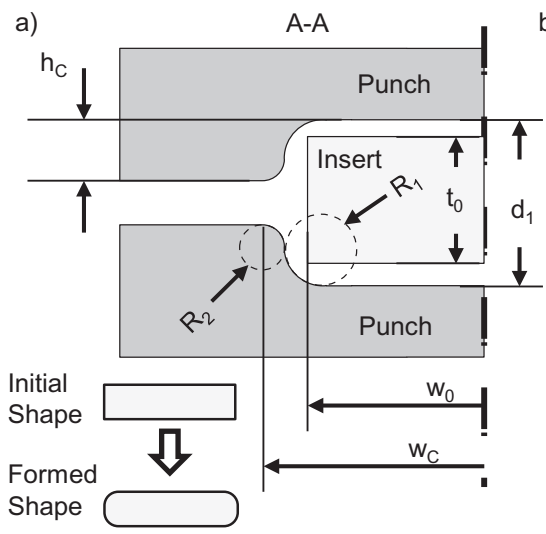

b)

\begin{tabular}{|l|l|}
\hline \multicolumn{2}{|c|}{ Varied Parameter } \\
\hline Radius $\mathrm{R}_{1}[\mathrm{~mm}]$ & $0.25 ; 0.35$ \\
\hline \multicolumn{2}{|c|}{ Constant Parameter } \\
\hline Radius $\mathrm{R}_{2}[\mathrm{~mm}]$ & 0.15 \\
\hline Height $\mathrm{h}_{\mathrm{C}}[\mathrm{mm}]$ & 0.40 \\
\hline Cavity $\mathrm{w}_{\mathrm{C}}[\mathrm{mm}]$ & 5.33 \\
\hline Initial Thickness $\mathrm{t}_{0}[\mathrm{~mm}]$ & 1.00 \\
\hline Initial Width $\mathrm{w}_{0}[\mathrm{~mm}]$ & 5.00 \\
\hline Min. Distance $\mathrm{d}_{1}[\mathrm{~mm}]$ & 1.00 \\
\hline
\end{tabular}

Figure 3. Schematic illustration of the edge rounding process (a) and geometrical parameters (b).

were used with constant orientation of the rolling direction as shown in Fig. 2c. The copper alloy was chosen as a representative material for electronic connectors whereas the aluminium alloy is associated with good forming and blanking properties and its resistance to corrosion in severe environment. The embossing process is basically considered as dry forming since specimens and tools were degreased so that no further cleaning would be needed before overmoulding.

The detailed shape of the punch is illustrated in Fig. 3. The essential aspects of the punch geometry are two radii. Radius $\mathrm{R}_{1}$ defines the edge geometry and thus, it is the point of interest. Radius $\mathrm{R}_{2}$ supports the centring and the orientation of the metal insert during the forming process. In order to ensure a smooth surface of the cavity the two radii are linked by a tangential condition. The height $\mathrm{h}_{\mathrm{C}}$ of the cavity as well as the distance $\mathrm{w}_{\mathrm{C}}$ between the radii $\mathrm{R}_{2}$ were kept constant for each set of punches. Due to the constant distance $\mathrm{w}_{\mathrm{C}}$ the position of the centres of $\mathrm{R}_{1}$ varied accordingly. This leads to a nominal cross section area of $1.975 \mathrm{~mm}^{2}$ for $\mathrm{R}_{1}=0.35 \mathrm{~mm}$ and slightly larger area of $1.995 \mathrm{~mm}^{2}$ for $\mathrm{R}_{1}=0.25 \mathrm{~mm}$. Furthermore, the open coining process allows the variation of the punch stroke and the remaining gap can compensate excessive material during forming.

\section{Effect of component tolerances on the edge rounding}

Since the edge rounding operation targets to be implemented in a progressive die the influence of the small size of the formed feature, the effect of workpiece tolerances and sheared edges on formed shape have to be analysed. Therefore, the sheet thickness $t_{0}$ was varied within the tolerance of $\pm 0.04 \mathrm{~mm}$ and 

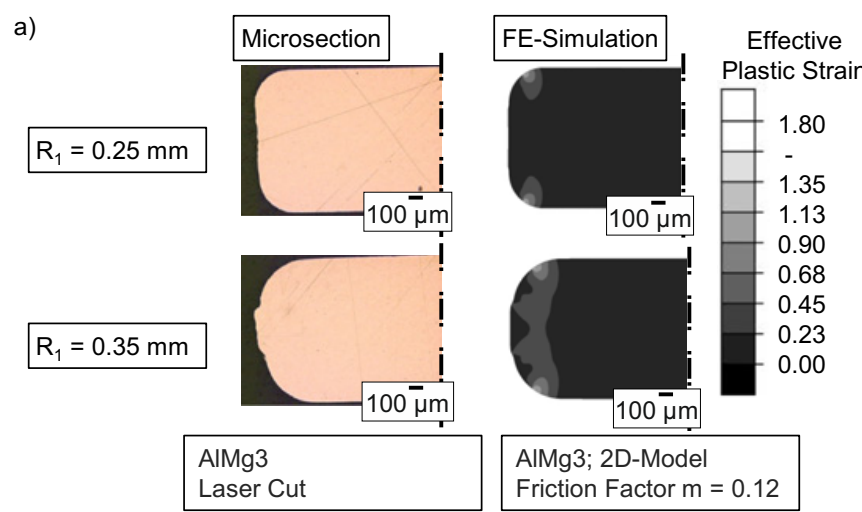

b)

\begin{tabular}{|l|l|l|}
\hline Material Data & AlMg3 & CuSn6 \\
\hline $\begin{array}{l}\text { Young's Modulus E } \\
{\left[\mathrm{N} / \mathrm{mm}^{2}\right]}\end{array}$ & $70 \mathrm{e}+3$ & $102 \mathrm{e}+3$ \\
\hline $\begin{array}{l}\text { Yield Strength YS } \\
{\left[\mathrm{N} / \mathrm{mm}^{2}\right]}\end{array}$ & 127 & 363 \\
\hline
\end{tabular}

c)

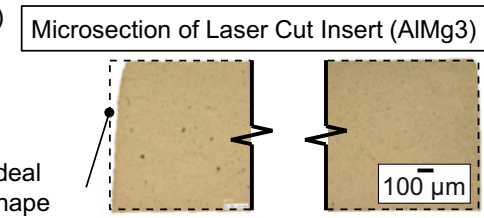

Figure 4. Comparison of experiment and FE-simulation for different edge radii (a), used material data (b) and microsection of laser cut insert compared to ideal shape (c).

the width $\mathrm{w}_{0}$ was changed in the range of $\pm 0.03 \mathrm{~mm}$ due to specifications of the assembly injection moulding process. Finite element simulation was used to investigate the influence of the variation of tolerances. Due to the small cross section compared to the length of the forming area plain strain condition could be assumed and thus the process was described by a $2 \mathrm{D}$-model with the software simufact.forming 12.0.1 ${ }^{\circledR}$. In order to ensure the exact modelling of small features an initial mesh size of $0.030 \mathrm{~mm}$ was chosen and additionally refinement boxes with a mesh size down to $0.003 \mathrm{~mm}$ were placed in the forming area. The friction factor model was used with $\mathrm{m}=0.12$ based on literature for similar materials and forming operations [7]. The material data shown in Fig. 4b were obtained by layer compression tests.

The investigation of influence of varying thickness and width was based on an ideal rectangular shape for simulation. In order to compare simulated and experimental results laser cut parts were used since their cross section is close to a rectangular shape according to Fig. 4c. As shown in Fig. 4a there is a good geometrical correlation between simulation and experiment. For $\mathrm{R}_{1}=0.35 \mathrm{~mm}$ a stronger impact on the strained volume can be observed and the smaller volume of the cavity of $\mathrm{R}_{1}=0.35 \mathrm{~mm}$ leads to a slight bulging compared to $\mathrm{R}_{1}=0.25 \mathrm{~mm}$. The impact of the variation of the dimensions according to the tolerance range was also investigated by FE-simulation. Thus, an ideal rectangle shaped cross section of the metal insert was assumed and varied in thickness and width between the lower and upper tolerance limits. From the results in Fig. 5 it can be concluded that the nominal dimension and the underfilling due to lower bound dimensions produce a desirable shape. In contrast, an overfilling leads to an excessive bulging which is critical in respect to the subsequent overmoulding process in terms of stress concentration. This behaviour is mainly shape dependent.

Since typically metal inserts are blanked the shape of the sheared edge is affected by the workpiece material. Experimental results show that for $\mathrm{CuSn6}$ the share of the burnished zone can reach $60 \%$ whereas for AlMg3 it is only $37 \%$. Thus the facture zone is significantly larger which in turn leads to a higher loss of material for $\mathrm{AlMg} 3$ during blanking. In order to simulate the impact of the sheared edge geometry the contour of the edge was derived of microsections of actual blanked parts. The gained contour data was used to model the cross section of the blanked part as input for the simulation. Since the investigations focused on geometrical aspects work hardening of the blanking was neglected. According to Fig. 5 it can be concluded that for blanked parts of CuSn6 as well as for AlMg3 a slight underfilling can be expected. For CuSn6 the final insert shape matches very well with the desired contour while for AlMg3 measures to compensate the material loss have to be considered. 


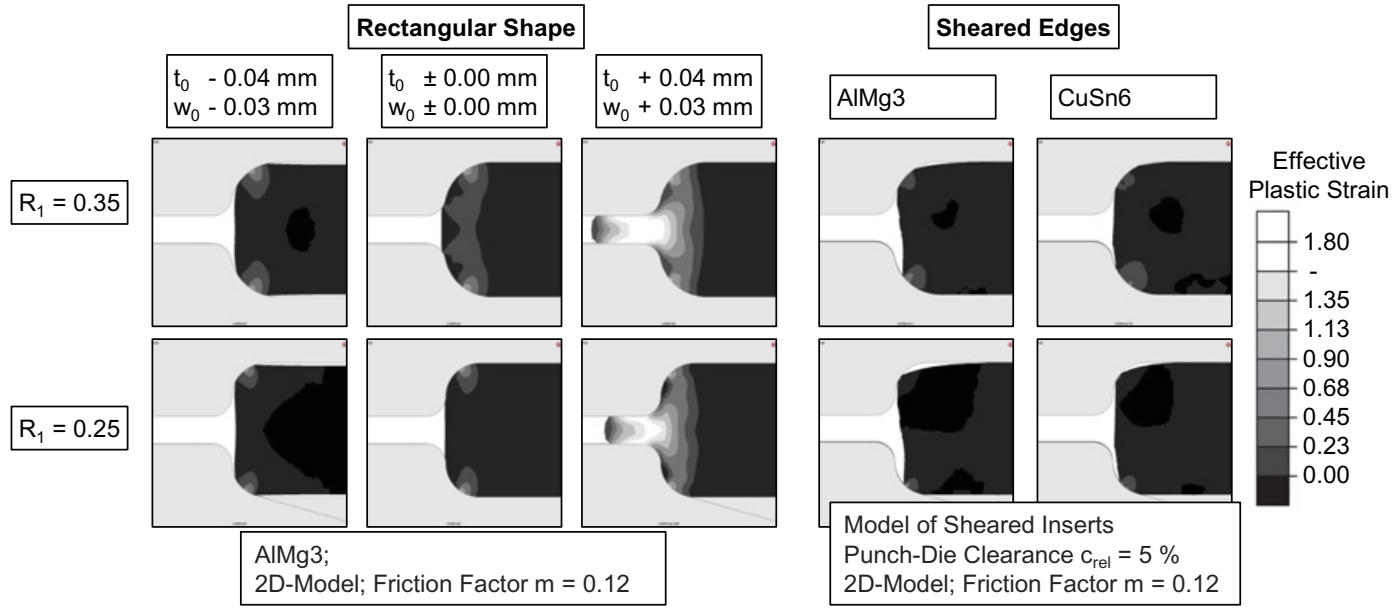

Figure 5. Influence of varying insert dimensions and sheared edges on the edge rounding.

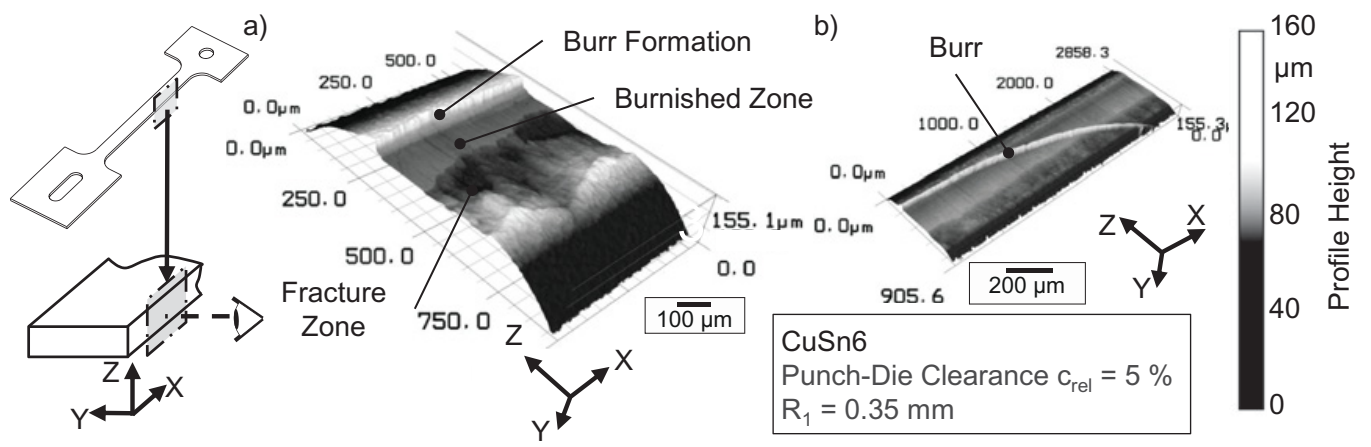

Figure 6. Measured 3D-geometry of burr formation (a) and burr (b) due to edge rounding.

\section{Burr formation during edge rounding}

The experiments also showed effects which were not anticipated by the simulation. In Fig. 6 the formation of a burr along the edge was observed. The pictures in Fig. 6 were taken with the laser scanning microscope Keyence VK-X200 and illustrate the burnished and the fracture zone of the blanking process combined with the material built-up of the edge forming. In contrast to the results of the simulation it seems that the upper punch scrapes material of the edge which is detached from the workpiece, Fig. 6b. Such loose or partly loose particles are undesired since they represent sharp artefacts and can possibly affect the bonding between the metal and the polymer if they break loose during the overmoulding process.

\section{Thermo-mechanical stress in polymer metal hybrid parts}

To gain qualitative information about the influence of the different edge radii and insert geometries on the polymer metal hybrid regarding stress concentration during temperature changes simulations were carried out. Therefore, calculated cross sections of the forming simulation for $\mathrm{R}_{1}=0.35 \mathrm{~mm}$ for a 


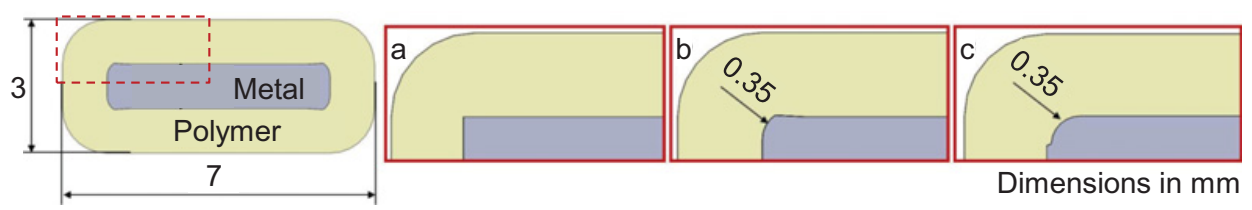

Figure 7. Cross sections for FE-simulation with different metal insert cross sections: a) reference without rounded edges, b) partially formed insert $R_{1}=0.35 \mathrm{~mm}, \mathrm{c}$ ) fully formed insert $\mathrm{R}_{1}=0.35 \mathrm{~mm}$.

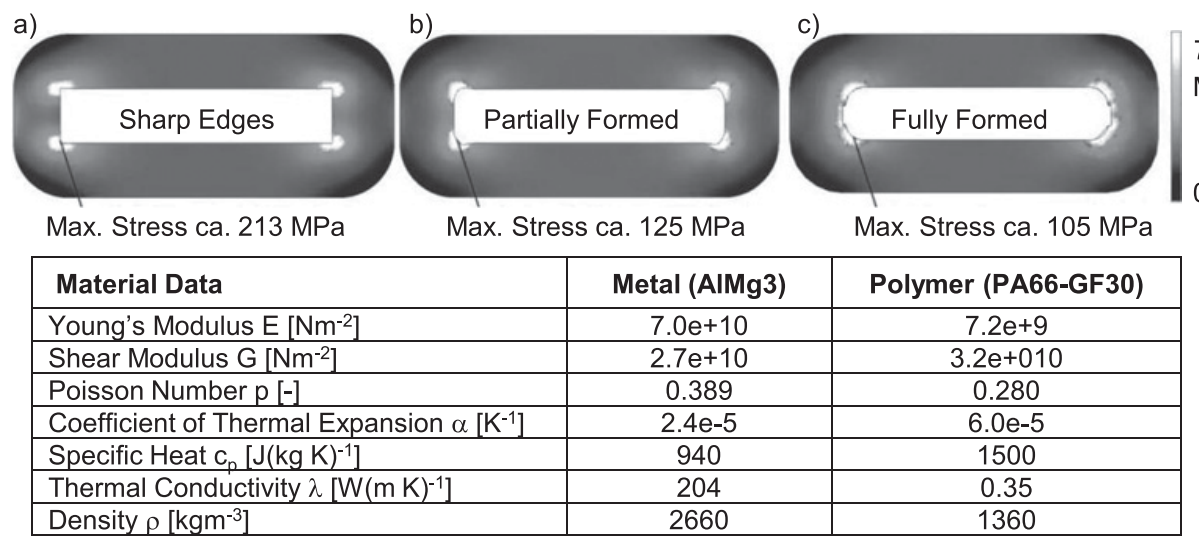

Figure 8. Stress in the polymer component of a polymer metal hybrid with different metal insert cross sections during cooling from $150^{\circ} \mathrm{C}$ to room temperature $\left(23^{\circ} \mathrm{C}\right)$, punch with $\mathrm{R} 1=0.35 \mathrm{~mm}$.

full and a partial punch stroke were used as input geometry for the FE-simulation of the polymer metal hybrid shown in Fig. 7. The partial formed insert was used to analyse the impact of an imperfect forming process.

The load of the $\mathrm{FE}$-simulation was a temperature drop from $150^{\circ} \mathrm{C}$ (zero elongation) to room temperature $\left(23^{\circ} \mathrm{C}\right)$. The material data is listed in Fig. 8. The polymer is Polyamide 66 with 30 weight-\% glass fibres (PA66-GF30, Ultramid A3EG6, BASF SE), a very common polymer for electronic applications. To simplify the model the data were assumed to be not temperature dependent, linear, isotropic and without consideration of relaxation. The stress distribution based on the results of the FE-simulation is illustrated in Fig. 8. It can be concluded that rounded edges reduces the stress in the polymer part.

\section{Summary}

In order to provide electronic components with long lasting media tightness in severe environment for automotive and industrial application overmoulding metal inserts with rounded edges is a promising approach. The present work shows that defined radii in the range of $0.25 \mathrm{~mm}$ to $0.35 \mathrm{~mm}$ can be realized by microforming. Furthermore it can be derived that variation of the insert dimension within the tolerance limits of the sheet thickness and the width of the blanked part leads to a distinct effect on the formed shape of the insert after the edge rounding. In addition, an influence of the workpiece material was observed due to material dependent cutting edge geometries for blanked parts. Based on thermo-mechanical FE-simulation it can be shown that the stress within the polymer can be reduced by half due to rounded edges compared to sharp edges. 


\section{ICNFT 2015}

The authors thank the German Research Foundation (DFG) for funding this study within the project ME 2043/ 33-1 and DR 421/7-1 "Fundamental investigation of blanking, forming and assembly injection moulding and their interdependence for manufacturing durable tight electronic systems".

\section{References}

[1] R. Chitkara, W. Ballhaus, B. Kliem, S. Berings, B. Weiss, Spotlight on Automotive - PwC Semiconductor Report (2013)

[2] C. Brecher (Eds.), Integrative Production Technology for High-Wage Countries, Springer, Berlin (2012)

[3] R. Isermann, Mechatronic systems - Fundamentals, Springer, London (2003)

[4] C. Heinle, M. Vetter, Z. Brocka-Krzeminska, G. W. Ehrenstein, D. Drummer, J. Plast. Technol. 6, $428-450$ (2009)

[5] M. Achouri, E. Gildemyn, G. Germain, P. Dal Santo, A. Potiron, Int J Adv Manuf Technol 5-8, 1019-1032 (2014)

[6] M. Geiger, M. Kleiner, R. Eckstein, N. Tiesler, U. Engel, Annals of the CIRP 2, 445-462 (2001)

[7] T. Stellin, U. Engel, M. Merklein in: M. Jun, S. Park (Eds.), Proc. 8th International Conference on MicroManufacturing (2013) 